CLINICAL STUDY

\title{
Do muscle fiber conduction slowing and decreased levels of circulating muscle proteins represent sensitive markers of steroid myopathy? A pilot study in Cushing's disease
}

\author{
Marco A Minetto ${ }^{1,2}$, Fabio Lanfranco ${ }^{1}$, Alberto Botter ${ }^{2}$, Giovanna Motta ${ }^{1}$, Giulio Mengozzi ${ }^{3}$, Roberta Giordano ${ }^{4}$, \\ Andreea $\mathrm{Picu}^{1}$, Ezio Ghigo ${ }^{1}$ and Emanuela Arvat ${ }^{1}$ \\ ${ }^{1}$ Division of Endocrinology, Diabetology and Metabolism, Department of Internal Medicine, Molinette Hospital, University of Turin, C.so Dogliotti 14, \\ 10126 Turin, Italy, ${ }^{2}$ Laboratory for Engineering of the Neuromuscular System (LISiN), Department of Electronics, Politecnico di Torino, 10129 Turin, \\ Italy, ${ }^{3}$ Clinical Chemistry Laboratory, Molinette Hospital, 10126 Turin, Italy and ${ }^{4}$ Department of Clinical and Biological Sciences, University of Turin, \\ 10043 Orbassano, Italy
}

(Correspondence should be addressed to M A Minetto at Division of Endocrinology, Diabetology and Metabolism, Department of Internal Medicine, Molinette Hospital, University of Turin; Email: marco.minetto@unito.it)

\begin{abstract}
Objective: Glucocorticoids are known to decrease protein synthesis and conduction velocity of muscle fibers. However, the degree of impairment of muscle protein synthesis and conduction slowing in patients with Cushing's disease remains poorly characterized. Our objective was to investigate whether and to what extent chronic endogenous hypercortisolism could decrease the circulating levels of muscle proteins and modify myoelectric indexes of sarcolemmal excitability and fatigability.

Design: A total of ten patients with Cushing's disease and 30 healthy controls matched for age, sex, and body mass index were compared.

Methods: Blood sampling and electrophysiological tests on vastus lateralis, vastus medialis, and tibialis anterior muscles were performed.

Results: Serum creatine kinase (CK) and plasma myoglobin were significantly lower in patients with respect to controls $(P<0.001$ and $P<0.05$ respectively): the mean relative difference between patients and controls was $48.9 \%$ for CK and $21.4 \%$ for myoglobin. Muscle fiber conduction velocity (MFCV) and myoelectric manifestations of fatigue were significantly decreased in all muscles of the patients with respect to controls. The mean relative difference in MFCV between patients and controls was $26.0 \%$ for vastus lateralis, $22.9 \%$ for vastus medialis, and $11.6 \%$ for tibialis anterior. These differences contrasted with the paucity of signs suggestive of myopathy that were obtained by needle electromyography in the patients. Conclusions: Slowing of muscle fiber conduction and decreased levels of circulating muscle proteins are sensitive markers of impaired muscle function, which are suitable for use in combination with clinical assessment and standard electrodiagnostic tests for accurate identification and follow-up of myopathic patients.
\end{abstract}

European Journal of Endocrinology 164 985-993

\section{Introduction}

Steroid myopathy is a well-known sign of Cushing's syndrome (1-3). It has a typical pattern of muscle weakness affecting the lower limbs more than the upper limbs and the proximal part of a limb more than the distal part (4). Although the cause of steroid myopathy is not precisely known, it appears that the major effect of the chronic glucocorticoid excess is to blunt muscle protein synthesis and activate muscle proteolysis (4). In subjects under glucocorticoid therapy, the downregulation of muscle protein synthesis can be unraveled by decreased levels of circulating muscle proteins (5). However, it may be hypothesized that the circulating levels of muscle proteins could potentially also be reduced in endogenous hypercortisolism. Apart from the preliminary observation of Khaleeli et al. (1) who found a plasma creatine kinase (CK) activity at the lower end of the normal range in two out of three patients with Cushing's disease, no previous study investigated whether the levels of circulating proteins such as CK and myoglobin are reduced in patients with Cushing's disease compared with healthy controls.

Furthermore, two in vivo studies also showed a slowing of the muscle fiber conduction following short-term glucocorticoid administration $(5,6)$ : it has been suggested that the decrease in muscle fiber conduction velocity (MFCV) was related to the suppressive effect of glucocorticoids on sarcolemmal excitability $(5,6)$. Besides sarcolemmal excitability, the other factor influencing MFCV is muscle fiber diameter. It may be hypothesized that both these factors are affected 
in the classic (chronic) form of steroid myopathy that occurs in endogenous hypercortisolism. However, the value of performing MFCV measurements in patients suspected of steroid myopathy has not been investigated.

In this study, blood samples were collected and electromyographic (EMG) signals were detected from three muscles of the lower limb in patients with Cushing's disease. This disorder and these muscles were specifically selected because the majority of the patients manifest relevant atrophy and weakness of limb muscles, whereas respiratory muscles are usually spared (7). The aim was to investigate whether and to what extent chronic endogenous hypercortisolism could decrease the circulating levels of muscle proteins and modify MFCV and myoelectric manifestations of fatigue.

\section{Materials and methods}

\section{Subjects}

A total of ten patients with Cushing's disease (seven women and three men) were studied. All patients had signs and symptoms of overt hypercortisolism and were evaluated in an active phase of the disease (urinary free cortisol $>150 \mu \mathrm{g} / 24 \mathrm{~h}$ in all patients).

The diagnosis of Cushing's disease was made according to the international criteria (8), including high daily urinary free cortisol, absent cortisol suppression after low-dose dexamethasone test $(>1.8 \mu \mathrm{g} / \mathrm{dl})$, and lack of the cortisol circadian rhythm (midnight cortisol $>7.5 \mu \mathrm{g} / \mathrm{dl}$ ); moreover, the diagnosis was based on the presence of normal or high levels of plasma ACTH and adequate cortisol suppression after a highdose dexamethasone test $(>80 \%)$. Furthermore, magnetic resonance was performed and showed a pituitary microadenoma in all patients. The presumed duration of disease ( $10.0 \pm 11.8$ years) was assessed by comparison of photographs and by patient interviews to date the onset of signs or symptoms that heralded a hypercortisolemic state. Impaired gonadal function was assumed in men in the presence of specific symptoms and testosterone levels $<3 \mathrm{ng} / \mathrm{ml}$, and in women in the presence of oligomenorrhea, or amenorrhea, or menopause (menopausal status was defined as "postmenopausal' if at least 1 year was elapsed since the last menstrual flow) and estradiol levels $<35 \mathrm{pg} / \mathrm{ml}$. At the time of diagnosis, two female patients were receiving statin treatment (simvastatin $20 \mathrm{mg} / \mathrm{die}$ ) due to longlasting alterations of serum cholesterol levels. Of these two patients, one was diabetic and required glucoselowering therapy (metformin $500 \mathrm{mg}$ b.i.d.). In addition, one male and one female patient had impaired fasting glucose and were receiving specific dietotherapy.

Although no direct measurements of lean body mass and force of anterior thigh/leg muscles were obtained in this study, all patients were categorized as frail based on the presence of three or more Fried's frailty criteria (9).
Furthermore, all patients presented clinical features related to lower extremity muscle weakness (such as inability to run or arise from low chairs) and rated an average fatigue severity scale (10) score (for the last 2 weeks prior to assessment) significantly greater than that of the healthy controls (see Results).

A total of 30 healthy subjects (20 women and ten men) served as controls. Health status was assessed by medical history, physical examination, blood count and chemistry, urinalysis, and electrocardiogram.

All the subjects received a detailed explanation of the study and gave written informed consent. The study conformed with the guidelines in the Declaration of Helsinki and was approved by the local ethics committee.

\section{Laboratory assays}

Fasting 0800 h blood samples were obtained. Serum CK activity was measured using the standardized 'reverse reaction' (creatine phosphate and ATP) and activation by $\mathrm{N}$-acetylcysteine. The analytical sensitivity of the method was $3 \mathrm{U} / \mathrm{l}$, and inter- and intra-assay coefficients of variation were below $2.4 \%$. Reference intervals for healthy people are 39-308 U/l for men and 26-192 U/l for women. Plasma myoglobin concentrations were determined by a sandwich electrochemiluminescent immunoassay, using two monoclonal myoglobin-specific antibodies. The lower limit of detection was $21 \mathrm{ng} / \mathrm{ml}$, and within-run and betweenrun precision assessments were $<3.4$ and $7.1 \%$ respectively. The expected values obtained on healthy subjects are $28-72 \mathrm{ng} / \mathrm{ml}$ for men and 25-58 ng/ml for women.

Serum and urinary cortisol were measured by RIA (Sorin Biomedica, Saluggia, Italy), plasma ACTH was measured by IRMA (Nichols Institute Diagnostics, San Juan Capistrano, CA, USA), serum estradiol was measured by RIA (Ultra-sensitive RIA DSL-4800, Pantec, Torino, Italy), and serum testosterone was measured by an automated chemiluminescence-based immunoassay with an ARCHITECT analyzer (Abbott). All hormone assays of an individual patient were performed in duplicate in the same assay session. Sera were immediately separated and stored at $-20{ }^{\circ} \mathrm{C}$ until assayed. Intra- and inter-assay coefficients of variation for all the above-mentioned hormonal assays were below 8 and $12 \%$ respectively.

\section{Electrophysiological tests, stimulation technique, and EMG recordings}

Non-invasive electrophysiological tests were performed, in both patients and controls, for the following muscles of the dominant side: vastus lateralis, vastus medialis, and tibialis anterior. These muscles were selected because they present differences in fiber-type composition: the percentages of type 2 fibers are $50 \%$ for the 
vasti and 30\% for the tibialis anterior (11). For each muscle, the following protocol was adopted: i) the subject's limb was placed in an isometric brace and the joint was fixed at $120^{\circ}\left(180^{\circ}\right.$ being full extension of the knee/ankle); ii) the muscle motor point was identified, using a stimulation pen electrode, as the location generating the maximal mechanical response with the minimum injected current $(5,12,13)$, and an adhesive stimulation electrode was placed over it; iii) an adhesive array of surface electrodes for detection of electrically elicited EMG signals (massed action potentials, M-waves) $(5,12,13)$ was located between the motor point and the distal tendon; and iv) one $60 \mathrm{~s}$ long stimulation burst was delivered at the current intensity generating the maximal M-wave (see below) and at the stimulation frequency of $20 \mathrm{~Hz}$.

Electrical stimulation was provided by a programmable stimulator (LISiN, Torino, Italy). An adhesive stimulation electrode $(30 \times 30 \mathrm{~mm}$; Spes Medica, Battipaglia, Italy) was placed over the motor point and a larger electrode $(50 \times 80 \mathrm{~mm})$ was placed over the antagonist muscle (monopolar stimulation) $(5,12,13)$. For each stimulation burst, biphasic rectangular pulses (200 $\mu$ s duration each) were delivered at the maximal current intensity identified as follows: M-waves were monitored as the muscle was stimulated at $2 \mathrm{~Hz}$ with current pulses of increasing intensity. The stimulation intensity was increased until the M-wave peak amplitude reached a plateau. The current corresponding to the maximal M-wave was identified as the current intensity of the M-wave amplitude plateau, followed by an absence of changes for a further increase of up to $10 \mathrm{~mA}(5,12,13)$.

The surface EMG signals were detected by a linear array of eight electrodes ( $5 \mathrm{~mm}$ inter-electrode distance; LISiN - Spes Medica, Italy) in single differential configuration. The optimal position and orientation of the array was searched and selected as that providing i) M-waves showing propagation from the motor point to the distal tendon and ii) the most similar M-wave shape in different channels. Before placement of the array, the skin was lightly abraded with abrasive paste. To ensure proper electrode-skin contact, $20 \mu \mathrm{l}$ conductive gel was inserted into the electrode cavities of the array with a gel dispenser. The bipolar surface EMG signals were amplified (16-channel surface EMG amplifier, LISiN), bandpass filtered (10-500 Hz), sampled at 2048 samples/s per channel, converted to digital data (12 bit A/D converter), and stored.

Needle EMG was performed, in the patient group only, for quantitative motor unit action potential (MUAP) analysis of the same three muscles studied by the surface EMG. As high levels of muscle contractions imply overlap of MUAPs of concurrently active motor units (i.e. loss of information that occurs when overlapping positive and negative phases of MUAPs cancel one another and reduce the amplitude of the signal), patients were asked to perform isometric lowforce contractions (intensity $<5 \%$ of the maximal force) following insertion of the recording electrode into the muscle. After the collection of several MUAPs of 4-6 motor units from the first insertion site, the needle was relocated to a different site for an additional 4-6 MUAPs. This process was repeated until MUAPs of 20 different motor units were recorded.

Intramuscular EMG signals were detected using a disposable concentric needle electrode (model 0028.013; Bionen, Firenze, Italy). Skin was cleaned with alcohol prior to insertion of the needle electrode. The bipolar intramuscular EMG signals were amplified (XCalibur; Excel-Tech, Toronto, ON, Canada), bandpass filtered $(5 \mathrm{~Hz}-10 \mathrm{kHz})$, sampled at $20 \mathrm{kHz}$, converted to digital data (12 bit A/D converter), and stored.

\section{Signal analysis}

Surface EMG variables of interest were MFCV and mean frequency of the power spectrum (Fig. 1 in Minetto et al. (5)).

MFCV, that is the velocity with which action potentials move along muscle fibers, is related to
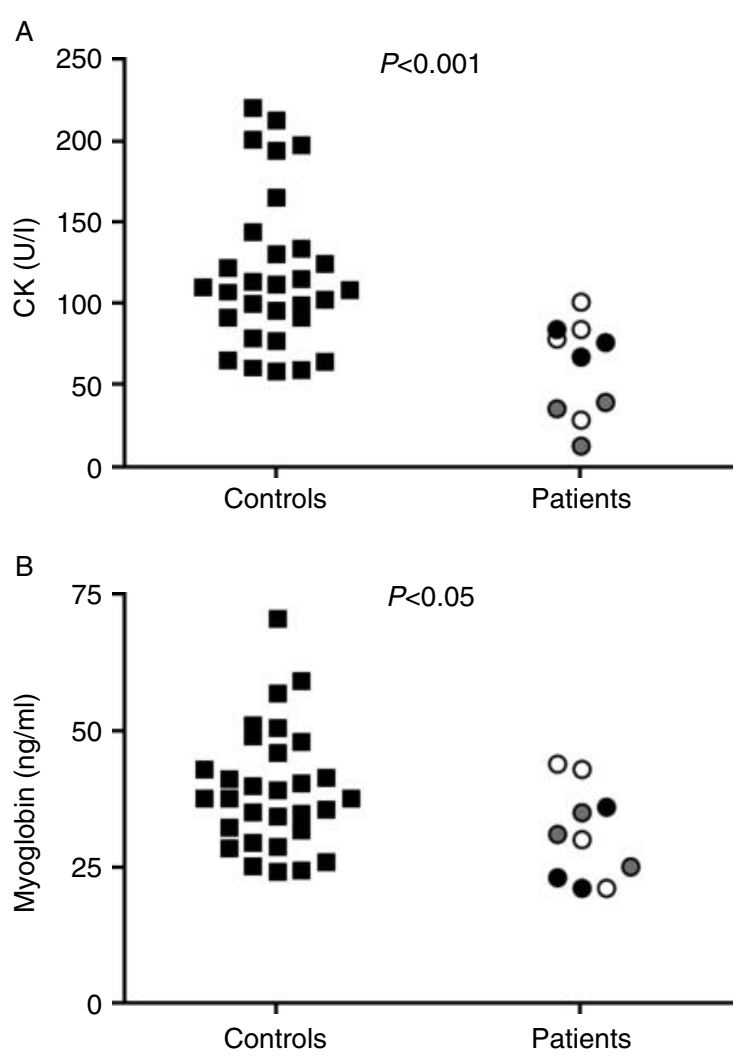

Figure 1 Serum creatine kinase (CK; Panel A) and plasma myoglobin levels (Panel B) in healthy controls (squares, $n=30$ ) and patients with Cushing's disease (circles, $n=10$ ). Significant differences are indicated by the respective $P$ values. Black and gray circles in both panels indicate CK and myoglobin values of six patients presenting myopathic MUAPs in the vastus medialis (black circles, $n=3$ ) and lateralis (gray circles, $n=3$ ) muscles. 
sarcolemmal excitability (14). In fact, the action potential propagation depends on the generation of the membrane potential and therefore on the concentration of essential ions on both sides of the membrane and the function of numerous ion channels, most importantly the sodium channels (15). Moreover, MFCV is related to the diameter of muscle fibers (as diameter determines the cytoplasmic resistance of a fiber) $(16,17)$. Physiological values of MFCV estimated from surface EMG signals detected during electrically elicited muscle contractions (i.e. the average conduction velocity of all the muscle fibers that are excited by the externally imposed electrical field) ranged from 4.0 to $6.0 \mathrm{~m} / \mathrm{s}$ for the vastus lateralis and medialis muscles $(5,13,18,19)$ and from 3.5 to $5.5 \mathrm{~m} / \mathrm{s}$ for the tibialis anterior muscle $(5,20,21)$.

Mean frequency provides some basic information about the spectrum of the surface EMG signal: during fatiguing contractions, a compression of the signal spectrum toward low frequencies (Fig. 1 in Minetto et al. (5)) was found to be associated with metabolic changes in the muscular microenvironment and adaptations of membrane properties of muscle fibers $(22,23)$.

The $20 \mathrm{M}$-waves corresponding to each epoch of $1 \mathrm{~s}$ were averaged, thereby obtaining, for each bipolar channel, 60 averaged M-waves during the $60 \mathrm{~s}$ long contraction (18). Thereafter, a sequence of 60 surface EMG variable estimates was calculated from three to seven bipolar channels (average \pm s.D. number of selected channels: $4.3 \pm 1.1$ ) with multichannel methods $(5,13)$ : channel selection was based on the criterion of a minimal change in the shape of $\mathrm{M}$-waves between consecutive channels. From the selected channels, the average of the first two MFCV estimates (first $2 \mathrm{~s}$ ) was considered for the determination of MFCV at the beginning of the contraction, whereas the whole group of 60 estimates of mean frequency (that were obtained, in each $1 \mathrm{~s}$ long epoch, as the average of all the channels selected for MFCV estimation) was considered to study myoelectric manifestations of fatigue. A regression line fitted the change in mean frequency estimates during the $60 \mathrm{~s}$ long contraction. The intercept of the regression line at time $=0$ was considered the initial value, and the slope of the line was used as an estimate of the rate of change over time. Normalized rate of change was defined as the slope divided by the initial value and expressed as a percent per unit time $(5,13)$. This normalization allowed the comparison of the relative changes between different subjects and groups.

Using the multi-MUAP technique described previously $(24,25)$, the MUAP waveforms of 20 motor units were obtained for each muscle from the needle EMG recordings. As a myopathic process results in brief polyphasic MUAPs (as the result of loss of muscle fibers and alteration in conductivity through the damaged motor units), the MUAP duration is usually used as an indicator of myopathy. Thus, the MUAP duration was estimated for each of the 20 MUAPs detected from the three muscles and the outlier method proposed by Stålberg et al. (26) was adopted for quantitative MUAP analysis. Briefly, the outlier method categorizes a muscle (as neuropathic or myopathic) by counting the number of outliers of the considered MUAP parameter(s) (i.e. MUAP duration in this study) from the set of the first 20 MUAPs that are detected from the muscle under study. A MUAP parameter value is considered to be an outlier if it is above or below the high and low outlier thresholds for that parameter respectively. A muscle for which there are three or more outlying values of the MUAP duration below the low (above the high) outlier threshold is categorized as myopathic (neuropathic). In the present analysis, the low and high outlier thresholds for the MUAP duration have been obtained from the tables of normative data $(24,27)$.

\section{Statistical analysis}

Normal distribution of the data was tested by the Shapiro-Wilk test: because data were not normally distributed, non-parametric tests were used. The MannWhitney $U$ test was used to compare clinical characteristics, values of circulating muscle proteins, and myoelectric signal variables between patients and controls. Kruskal-Wallis ANOVA followed by Dunn's multiple comparison test was used for comparing MFCV estimates among the three muscles in each of the two subject groups. The Spearman analysis was used to test for correlations between estimated duration of disease and levels of circulating muscle proteins or estimates of myoelectric signal variables.

The level of statistical significance was set at $P=0.05$. The values are expressed as mean \pm s.D.

\section{Results}

\section{Clinical characteristics and laboratory assays}

The two groups of subjects were comparable for age, anthropometric measurements, and gonadal status (Table 1): no male subject was hypogonadic in the two groups, whereas two female patients and four female controls were postmenopausal. Testosterone levels in the three male patients and estradiol levels in the five non-menopausal female patients were lower (although non-significantly) than those of the healthy controls. The severity of fatigue reported by patients was significantly greater than that reported by the controls.

Serum CK was significantly $(P<0.001)$ lower in patients $(60.4 \pm 29.5 \mathrm{U} / \mathrm{l})$ with respect to control subjects $(118.3 \pm 47.2 \mathrm{U} / \mathrm{l})$ : the mean relative difference between patients and controls was $48.9 \%$. In one out of seven female patients, CK levels were below the lower limit of normal (26 U/l) (Fig. 2A). 
Table 1 Average ( \pm s.D.) values of age, anthropometric measurements, and gonadal status for the two groups of patients with Cushing's disease $(n=10)$ and healthy controls $(n=30)$.

\begin{tabular}{lccl}
\hline Variable & Patients & Controls & $\boldsymbol{P}$ value \\
\hline Age (years) & $50.1 \pm 10.5$ & $53.0 \pm 6.0$ & $\mathrm{NS}$ \\
Body mass $(\mathrm{kg})$ & $69.2 \pm 18.6$ & $74.0 \pm 12.3$ & $\mathrm{NS}$ \\
Height $(\mathrm{m})$ & $1.62 \pm 0.10$ & $1.66 \pm 0.10$ & $\mathrm{NS}$ \\
$\mathrm{BMl}\left(\mathrm{kg} / \mathrm{m}^{2}\right)$ & $26.1 \pm 5.2$ & $24.0 \pm 6.5$ & $\mathrm{NS}$ \\
Testosterone & $3.8 \pm 0.5^{\mathrm{a}}$ & $4.8 \pm 1.3^{\mathrm{a}}$ & $\mathrm{NS}$ \\
$\quad(\mathrm{ng} / \mathrm{ml})$ & & & \\
Estradiol (pg/ml) & $65.2 \pm 25.7^{\mathrm{b}}$ & $80.7 \pm 33.0^{\mathrm{b}}$ & $\mathrm{NS}$ \\
Fatigue severity & $5.6 \pm 0.4$ & $2.6 \pm 0.5$ & $<0.001$ \\
$\quad$ scale score & & &
\end{tabular}

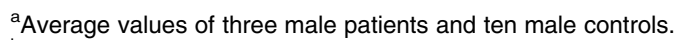

${ }^{\mathrm{b}}$ Average values of five non-menopausal female patients and 16 nonmenopausal female controls.

Plasma myoglobin was significantly $(P<0.05)$ lower in patients $(30.9 \pm 8.5 \mathrm{ng} / \mathrm{ml})$ with respect to control subjects $(39.3 \pm 11.1 \mathrm{ng} / \mathrm{ml})$ : the mean relative difference between patients and controls was $21.4 \%$. In two of the seven female patients, myoglobin levels were below the lower limit of normal $(25 \mathrm{ng} / \mathrm{ml})$ and corresponded to the minimum detectable concentration of $21 \mathrm{ng} / \mathrm{ml}$. In one of the three male patients, the myoglobin levels were below the lower limit of normal $(28 \mathrm{ng} / \mathrm{ml})$ (Fig. 2B).

A weak (not significant) negative correlation was observed between the estimated duration of disease and the CK levels ( $r=-0.6, P=0.07)$ : that is, the longer the estimated duration of disease, the lower the CK levels tended to be.

\section{MFCV and myoelectric manifestations of fatigue}

MFCV was significantly decreased in all muscles of the patients with respect to control subjects (Table 2). The mean relative differences between patients and controls was $26.0 \%$ for vastus lateralis (nine of the ten patients had values of MFCV $<4 \mathrm{~m} / \mathrm{s}$, whereas all control subjects had values $>4 \mathrm{~m} / \mathrm{s}$ ), $22.9 \%$ for vastus medialis (nine of the ten patients had values of MFCV $<4 \mathrm{~m} / \mathrm{s}$, whereas 29 of the 30 control subjects had values $>4 \mathrm{~m} / \mathrm{s}$ ), and $11.6 \%$ for tibialis anterior (eight of the ten patients had values of MFCV $<4 \mathrm{~m} / \mathrm{s}$, whereas 26 of the 30 control subjects had values $\geq 4 \mathrm{~m} / \mathrm{s}$ ).

The MFCV estimates of the vastus lateralis and medialis were significantly greater $(P<0.001$ and $P<0.05$ respectively) than those of the tibialis anterior in the control subjects, whereas no significant differences among the MFCV estimates of the three muscles were observed in the patient group.

Weak negative (although not significant: $r=-0.4$ $\pm 0.2, P=0.2$ ) correlations were observed in the patient group between estimated duration of disease and MFCV estimates of the three muscles: that is, the longer was the estimated duration of disease, the lower the muscle fiber conduction tended to be.

Figure 2 shows examples of M-wave time evolution (over $60 \mathrm{~s}$ of stimulation) for the vastus medialis muscle of one control subject (panel A) and one patient (panel B). Widening of the signal and decrease in amplitude during the contraction is evident in the control subject, whereas minor scaling in time and amplitude is evident in the patient. Accordingly, the normalized rate of change of mean frequency was greater (in absolute value) in the control subject $(-1.3 \% / \mathrm{s})$ with respect to the patient $(-0.8 \% / \mathrm{s})$. Similar to the comparison between these two subjects, significant differences between patients and controls were observed for all the three muscles (Fig. 2, panel E): the mean relative difference between patients and controls was $37.0 \%$ for
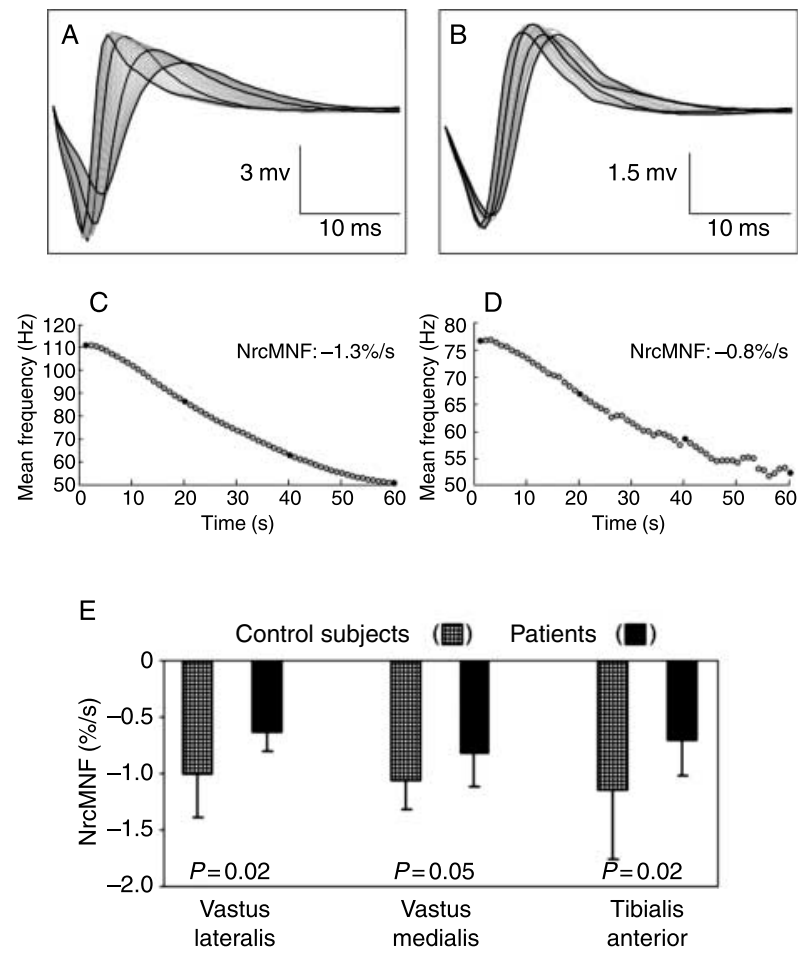

Figure 2 Examples of M-wave time evolution (over $60 \mathrm{~s}$ of stimulation) for the vastus medialis muscle of one healthy control $(A)$ and one patient $(B)$ of the same gender and age. Each curve is the average of the 20 responses obtained during $1 \mathrm{~s}$. Curves are $2 \mathrm{~s}$ apart. The first, twentieth, fortieth, and last (averaged) M-waves are reported in black color. (C and D) Time course of the mean frequency of the power spectrum throughout the $60 \mathrm{~s}$ long contraction for the two subjects of panels $\mathrm{A}$ and $\mathrm{B}$ : normalized rate of change of mean frequency (NrcMNF) is reported. Black circles indicate the value of mean frequency estimated for the first, twentieth, fortieth, and last (averaged) M-waves. (E) Normalized rates of change of mean frequency (NrcMNF) in control subjects $(n=30)$ and patients $(n=10)$ for the three studied muscles (vastus lateralis, vastus medialis, and tibialis anterior). Significant differences are indicated by the respective $P$ values. Mean values and S.D. bars are reported. 
Table 2 Estimates of MFCV in the three muscles investigated in the two groups of patients with Cushing's disease $(n=10)$ and healthy controls $(n=30)$.

\begin{tabular}{lccc}
\hline & \multicolumn{2}{c}{$\begin{array}{c}\text { MFCV estimates }(\mathrm{m} / \mathrm{s}) \\
\text { mean } \pm \text { s.D. }(\text { range })\end{array}$} & \\
\cline { 2 - 3 } Muscle & Patients & Controls & P value \\
\cline { 2 - 3 } & $3.7 \pm 0.6(3.0-4.6)$ & $5.0 \pm 0.5(4.3-5.9)$ & $<0.001$ \\
Vastus lateralis & $3.7 \pm 0.4(3.1-4.1)$ & $4.8 \pm 0.5(3.7-5.9)$ & $<0.001$ \\
Vastus medialis & $3.8 \pm 0.4(3.2-4.5)$ & $4.3 \pm 0.6(3.5-4.9)$ & $<0.05$ \\
Tibialis anterior & $3.8 \pm 0.4$ & \\
\hline
\end{tabular}

vastus lateralis, $22.0 \%$ for vastus medialis, and $38.6 \%$ for tibialis anterior.

A significant positive correlation was observed in the patient group between estimated duration of disease and normalized rate of change of the mean frequency for the tibialis anterior muscle $(r=0.7, P=0.05)$ : that is, the longer was the estimated duration of disease, the higher was the functional impairment of the tibialis anterior muscle.

\section{Needle EMG findings}

No evidence of brief MUAPs or other findings suggestive for myopathy, such as increased MUAP complexity (i.e. increased phases, turns, or linked components) or reduced area-to-amplitude ratio (i.e. the 'thinning' of the main spikes of the MUAP) (28), was obtained from visual inspection of the needle EMG recordings.

Quantitative MUAP analysis categorized three vastus lateralis muscles and three vastus medialis muscles (of different patients) as myopathic.

Average ( \pm s.D.) and range durations of myopathic MUAPs $(n=12)$ in the vastus lateralis muscle of the three patients were $7.9( \pm 1.4) \mathrm{ms}$ and $6.0-10.5 \mathrm{~ms}$ : the average value of the percent difference between duration of myopathic MUAPs and normative values of MUAP duration $(24,27)$ was $33.8 \pm 11.5 \%$. The estimated duration of disease in these three patients was $22 \pm 7$ years. All the three patients presented decreased levels of circulating muscle proteins (gray circles in Fig. 1: CK levels in the range 12-39 U/l; myoglobin levels in the range $25-31 \mathrm{ng} / \mathrm{ml}$ ), and muscle fiber conduction slowing (MFCV in the range 3.0-3.2 $\mathrm{m} / \mathrm{s}$ ).

Average ( \pm s.D.) and range durations of myopathic MUAPs $(n=13)$ in the vastus medialis muscle of the three patients were $7.0( \pm 0.9) \mathrm{ms}$ and $6.0-8.5 \mathrm{~ms}$ : the average value of the percent difference between duration of myopathic MUAPs and normative values of MUAP duration $(24,27)$ was $23.3 \pm 10.5 \%$. The estimated duration of disease in these three patients was $15 \pm 4$ years. All the three patients presented decreased levels of circulating muscle proteins (black circles in Fig. 1: CK levels in the range 67-84 U/l, myoglobin levels in the range $21-36 \mathrm{ng} / \mathrm{ml}$ ), and muscle fiber conduction slowing (MFCV in the range 3.4-3.9 m/s).

\section{Discussion}

The original findings of this study were the demonstrations of significant differences in circulating levels of muscle proteins, MFCV, and myoelectric manifestations of fatigue between patients with Cushing's disease and sex-, age-, and body mass index-matched controls. These differences contrasted with the paucity of signs suggestive for myopathy that were obtained through the standard electrodiagnostic investigation performed in the patients.

The paucity of electrophysiological signs is understandable given the limitation of the needle EMG examination that preferentially studies the type 1 muscle fibers $(4,29)$ and the histopathological finding of preferential type 2 muscle fiber impairment that occurs in steroid myopathy $(1,4)$. During a voluntary contraction, the first recruited motor units are composed of type 1 fibers (30). Because these fibers are not affected (as severely) as type 2 fibers in patients with steroid myopathy, there is little electrophysiological dysfunction to observe. If the patient is asked to increase the contraction force to also recruit motor units composed of type 2 fibers, abnormalities could occur but may not be observed as when type 2 fibers are recruited, too many motor units composed of type 1 fibers are concurrently firing, creating MUAP overlap and the consequent signal cancellation. This precludes quantitative assessment of any type 2 MUAP parameters in detail, thus limiting the sensitivity of the needle examination in diagnosing a preferential type 2 fiber myopathy (4). An additional factor that limits the needle EMG sensitivity in diagnosing a preferential type 2 fiber myopathy affecting the anterior thigh muscles is represented by the motor unit distribution in the vasti: within these muscles, a non-random arrangement of fiber types exists with the deeper portions of the muscles (those preferentially investigated by needle EMG) having more type 1 fibers than the more superficial portions $(31,32)$. Only when the myopathic disorder is severe enough to compromise type 1 fibers, abnormalities may be observed by needle EMG. Consistently, the quantitative MUAP analysis we adopted categorized as myopathic the vastus lateralis and medialis muscles of six different patients with an estimated long duration of disease and presenting severe muscle weakness, with an inability to climb stairs and arise from low chairs. Overall, the low sensitivity of the quantitative MUAP analysis we adopted is consistent with most characterizations of steroid myopathy that are based on qualitative visual and auditory analysis of EMG signals $(1,4)$ or different quantitative approaches (4). For example, it has been proposed to increase the number of parameters required to categorize a muscle as abnormal (including some or all of the following MUAP parameters: duration, amplitude, number of phases, number of turns, spike duration, and area-to-amplitude ratio) or to combine the outlier method proposed by Stålberg et al. (26) with the mean method proposed by 
Stewart et al. (33). However, these quantitative EMG approaches increase specificity at the expense of reduced sensitivity. Alternatively, interference pattern analysis methods $(34,35)$ or the recently proposed Bayesian muscle characterization (36) can be adopted for the analysis of needle EMG recordings. However, as the needle EMG is typically performed at low-force contraction levels, the estimates of MUAP features that can be obtained by the former and the latter methods are biased by slow motor units.

Regardless of the quantitative method that can be adopted for needle EMG analysis, our results indicate that the combination of needle EMG findings with clinical assessment, assays of circulating muscle proteins, and non-invasive estimation of MFCV and myoelectric fatigue profile seems to be a promising approach for an accurate detection of alteration of muscle fiber properties underlying steroid myopathy. Consistently, Blijham et al. (37) have found in patients with inflammatory myopathy (polymyositis, dermatomyositis, and inclusion body myositis) that the diagnostic accuracy of the needle EMG examination can be increased if it is combined with MFCV measurement.

The differences in CK and myoglobin levels we observed between patients and controls are related to the well-known anti-anabolic actions of glucocorticoids. This result is in line with the above-mentioned observation of Khaleeli et al. (1) as well as with the differences in circulating muscle proteins that were observed by Weber et al. (38) while comparing cachectic cancer patients and healthy controls. Interestingly, we found a greater difference between patients and controls in CK than in myoglobin levels: this could reflect the preferential impairment of type 2 fibers, which present high levels of CK expression, whereas myoglobin is preferentially expressed in oxidative type 1 fibers that are less affected by the myopathic process.

Also the differences in MFCV and myoelectric fatigue we observed between the two groups can be accounted for the glucocorticoid-induced preferential impairment $(5,6)$ and/or atrophy $(1,4)$ of (muscles mainly composed of) fast fibers. In fact, reductions in MFCV and myoelectric fatigue usually occur in elderly subjects (39) due to agerelated sarcopenia, which mainly consists in preferential loss and/or atrophy of type 2 fibers (40). We also recently found in healthy subjects that short-term glucocorticoid administration decreased MFCV and myoelectric manifestations of fatigue (5). Briefly, the higher is the functional impairment and/or the lower are the number and diameter (i.e. the higher is the degree of atrophy) of fast, fatigable (type 2) muscle fibers, the lower are the MFCV and myoelectric manifestations of fatigue. Consistently, in the patient group, we found no significant differences in MFCV estimates between vasti and tibialis anterior, whereas in the control subjects, the vastus lateralis and medialis muscles (that present a percentage of fast fibers around 50\%) had a greater MFCV than the tibialis anterior (that presents a percentage of fast fibers around 30\%). Although no relationship has been observed in animal and human models of steroid myopathy between duration of glucocorticoid administration and occurrence of myopathy (3), in this study, we found weak correlations between the estimated duration of disease and the decrease in CK levels and MFCV estimates. Accordingly, it may be hypothesized that after the onset of the myopathic process, muscle atrophy represents the cumulative, long-term consequence of the impaired balance of anabolic and catabolic mechanisms. It is presently not established how much of the impaired balance is due to the direct anti-anabolic and procatabolic actions of glucocorticoids on muscle cells versus the glucocorticoid-dependent impairment of the pituitary-gonadal axis and down-regulation of the muscular expression of the androgen receptor (41). Although in this study gonadal status was comparable between the two groups, patients tended to have lower values of gonadal hormones. Thus, it may be hypothesized that the differences in circulating levels of muscle proteins, MFCV, and myoelectric fatigue we observed between the two groups are partly accounted for by the differences in gonadal function. It also remains to be established whether the impaired balance of anabolic and catabolic processes and the consequent muscle atrophy can be reversed after correction of the hormonal disorder or drug withdrawal.

In conclusion, muscle fiber conduction slowing and decreased levels of circulating muscle proteins seem to be sensitive markers of steroid myopathy, which are suitable to be used in combination with clinical assessment and standard electrodiagnostic tests for accurate identification of myopathic patients and for monitoring the restoration of a normal muscle function after treatment.

\section{Declaration of interest}

The authors declare that there is no conflict of interest that could be perceived as prejudicing the impartiality of the research reported.

\section{Funding}

This study was supported by Regional Health Administration Project 'Ricerca Sanitaria Finalizzata 2009' and by bank foundations 'Compagnia di San Paolo' (Project 'Neuromuscular Investigation and Conditioning in Endocrine Myopathy'), 'Fondazione Cariplo' (Project 'Steroid myopathy: Molecular, Histopathological, and Electrophysiological Characterization'), and 'Fondazione Cassa di Risparmio di Saluzzo'.

\section{Acknowledgements}

Anonymous reviewers made many helpful contributions to this manuscript. The authors are grateful to Prof. R Merletti (LISiN, Politecnico di Torino, Italy) and Dr A Holobar (University of Maribor, Slovenia) for their careful review of the final version of the manuscript and to Dr I Girolami (School of Motor Sciences, Turin, Italy), Dr R Berardelli, Dr W Troni, and Dr E Berra (University of Turin, Italy) for their valuable assistance in subject recruitment and evaluation. 


\section{References}

1 Khaleeli AA, Edwards RH, Gohil K, McPhail G, Rennie MJ, Round J \& Ross EJ. Corticosteroid myopathy: a clinical and pathological study. Clinical Endocrinology 198318 155-166. (doi:10.1111/j. 1365-2265.1983.tb03198.x)

2 Kanda F, Okuda S, Matsushita T, Takatani K, Kimura KI \& Chihara K. Steroid myopathy: pathogenesis and effects of growth hormone and insulin-like growth factor-I administration. Hormone Research 200156 24-28. (doi:10.1159/000048130)

3 Owczarek J, Jasinska M \& Orszulak-Michalak D. Drug-induced myopathies. An overview of the possible mechanisms. Pharmacological Reports $2005 \mathbf{5 7} 23-34$.

4 Dumitru D. Myopathies. In Electrodiagnostic Medicine, 1st edn, pp 1031-1129. Ed. D Dumitru. Philadelphia: Hanley \& Belfus, 1995.

5 Minetto MA. Botter A. Lanfranco F, Baldi M, Ghigo E \& Arvat E. Muscle fiber conduction slowing and decreased levels of circulating muscle proteins after short-term dexamethasone administration in healthy subjects. Journal of Clinical Endocrinology and Metabolism 201095 1663-1671. (doi:10.1210/jc.2009-2161)

6 van der Hoeven JH. Decline of muscle fiber conduction velocity during short-term high-dose methylprednisolone therapy. Muscle and Nerve 199619 100-102. (doi:10.1002/(SICI)1097-4598 (199601)19:1 < 100::AID-MUS16 > 3.0.CO;2-)

7 Mills GH, Kyroussis D, Jenkins P, Hamnegard CH, Polkey MI, Wass J, Besser GM, Moxham J \& Green M. Respiratory muscle strength in Cushing's syndrome. American Journal of Respiratory and Critical Care Medicine 1999160 1762-1765.

8 Arnaldi G, Angeli A, Atkinson AB, Bertagna X, Cavagnini F, Chrousos GP, Fava GA, Findling JW, Gaillard RC, Grossman AB, Kola B, Lacroix A, Mancini T, Mantero F, Newell-Price J, Nieman LK, Sonino N, Vance ML, Giustina A \& Boscaro M. Diagnosis and complications of Cushing's syndrome: a consensus statement. Journal of Clinical Endocrinology and Metabolism 2003 88 5593-5602. (doi:10.1210/jc.2003-030871)

9 Fried LP, Tangen CM, Walston J, Newman AB, Hirsch C, Gottdiener J, Seeman T, Tracy R, Kop WJ, Burke G \& McBurnie MA. Frailty in older adults: evidence for a phenotype. Journals of Gerontology. Series A, Biological Sciences and Medical Sciences 200156 M146-M156.

10 Krupp LB, LaRocca NG, Muir-Nash J \& Steinberg AD. The fatigue severity scale. Application to patients with multiple sclerosis and systemic lupus erythematosus. Archives of Neurology $1989 \mathbf{4 6}$ 1121-1123.

11 Single-joint system function. In Neuromechanics of Human Movement, 3rd edn, pp 241-321. Ed. RM Enoka. Champaign: Human Kinetics, 2002.

12 Farina D, Blanchietti A, Pozzo M \& Merletti R. M-wave properties during progressive motor unit activation by transcutaneous stimulation. Journal of Applied Physiology 200497 545-555. (doi:10.1152/japplphysiol.00064.2004)

13 Botter A, Lanfranco F, Merletti R \& Minetto MA. Myoelectric fatigue profiles during electrically-elicited contractions of vastus lateralis, vastus medialis obliquus, and vastus medialis longus muscles. International Journal of Sports Medicine $2009 \mathbf{3 0}$ 408-417. (doi:10.1055/s-0028-1112142)

14 Andreassen S \& Arendt-Nielsen L. Muscle fibre conduction velocity in motor units of the human anterior tibial muscle: a new size principle parameter. Journal of Physiology 1987391 $561-571$

15 Blijham PJ, Drost G, Stegeman DF \& Zwarts MJ. Reduced musclefiber conduction but normal slowing after cold exposure in paramyotonia congenita. Muscle and Nerve 200837 23-26. (doi:10.1002/mus.20885)

16 Nandedkar SD \& Stålberg E. Simulation of single muscle fibre action potentials. Medical and Biological Engineering and Computing 198321 158-165. (doi:10.1007/BF02441531)

17 Blijham PJ, ter Laak HJ, Schelhaas HJ, van Engelen BG, Stegeman DF \& Zwarts MJ. Relation between muscle fiber conduction velocity and fiber size in neuromuscular disorders. Journal of Applied Physiology 2006100 1837-1841. (doi:10. 1152/japplphysiol.01009.2005)

18 Merletti R, Fiorito A, Lo Conte LR \& Cisari C. Repeatability of electrically evoked EMG signals in the human vastus medialis muscle. Muscle and Nerve 199821 184-193. (doi:10.1002/ (SICI)1097-4598(199802)21:2<184::AID-MUS5 > 3.0.CO;2-7)

19 Rainoldi A, Gazzoni M, Merletti R \& Minetto MA. Mechanical and EMG responses of the vastus lateralis and changes in biochemical variables to isokinetic exercise in endurance and power athletes. Journal of Sports Sciences 200826 321-331. (doi:10.1080/ 02640410701474499)

20 Knaflitz M, Merletti R \& De Luca CJ. Inference of motor unit recruitment order in voluntary and electrically elicited contractions. Journal of Applied Physiology 199068 1657-1667.

21 Kamavuako EN \& Farina D. Time-dependent effects of preconditioning activation on muscle fiber conduction velocity and twitch torque. Muscle \& Nerve 201042 547-555. (doi:10.1002/ mus.21726)

22 Brody LR, Pollock MT, Roy SH, De Luca CJ \& Celli B. pH-induced effects on median frequency and conduction velocity of the myoelectric signal. Journal of Applied Physiology 199171 1878-1885.

23 Kupa EJ, Roy SH, Kandarian SC \& De Luca CJ. Effects of muscle fiber type and size on EMG median frequency and conduction velocity. Journal of Applied Physiology 199579 23-32.

24 Bischoff C. Stålberg E, Falck B \& Eeg-Olofsson KE. Reference values of motor unit action potentials obtained with multi-MUAP analysis. Muscle and Nerve 199417 842-851. (doi:10.1002/ mus.880170803)

25 Stålberg E, Falck B, Sonoo M, Stålberg S \& Aström M. Multi-MUP EMG analysis - a two year experience in daily clinical work. Electroencephalography and Clinical Neurophysiology $1995 \mathbf{9 7}$ 145-154. (doi:10.1016/0924-980X(95)00007-8)

26 Stålberg E, Bischoff C \& Falck B. Outliers, a way to detect abnormality in quantitative EMG. Muscle and Nerve $1994 \mathbf{1 7}$ 392-399. (doi:10.1002/mus.880170406)

27 Motor unit potential studies. In Laboratory Reference for Clinical Neurophysiology, pp 418-431. Eds JA Livenson \& MM Dong. New York: Oxford University Press, 1992.

28 Nandedkar SD, Barkhaus PE, Sanders DB \& Stalberg EV. Analysis of amplitude and area of concentric needle EMG motor unit action potentials. Electroencephalography and Clinical Neurophysiology 198869 561-567. (doi:10.1016/0013-4694(88)90168-X)

29 Ramani V, Storey JR \& Lava NS. Electrophysiological testing in neurological disorders: electroencephalography, evoked potentials, and electromyography. In $A$ Guide to the Primary Care of Neurological Disorders, pp 87-97. Eds JA Popp \& BI Tramer, New York: Thieme, 1998.

30 Henneman E, Somjen G \& Carpenter DO. Functional significance of cell size in spinal motoneurons. Journal of Neurophysiology 1965 28 560-580.

31 Lexell J. Henriksson-Larsén K \& Sjöström M. Distribution of different fibre types in human skeletal muscles. 2. A study of cross-sections of whole m. vastus lateralis. Acta Physiologica Scandinavica 1983117 115-122. (doi:10.1111/j.1748-1716. 1983.tb07185.x)

32 Travnik L, Pernus F \& Erzen I. Histochemical and morphometric characteristics of the normal human vastus medialis longus and vastus medialis obliquus muscles. Journal of Anatomy $1995 \mathbf{1 8 7}$ 403-411.

33 Stewart CR, Nandedkar SD, Massey JM, Gilchrist JM, Barkhaus PE \& Sanders DB. Evaluation of an automatic method of measuring features of motor unit action potentials. Muscle and Nerve 198912 141-148. (doi:10.1002/mus.880120209)

34 Fuglsang-Frederiksen A. The utility of interference pattern analysis. Muscle and Nerve 200023 18-36. (doi:10.1002/(SICI) 1097-4598(200001)23:1<18::AID-MUS4 > 3.0.CO;2-B)

35 Finsterer J. EMG-interference pattern analysis. Journal of Electromyography and Kinesiology 200111 231-246. (doi:10.1016/ S1050-6411(01)00006-2) 
36 Pino LJ, Stashuk DW, Boe SG \& Doherty TJ. Probabilistic muscle characterization using QEMG: application to neuropathic muscle. Muscle and Nerve 201041 18-31. (doi:10.1002/mus. 21456)

37 Blijham PJ, Hengstman GJ, Ter Laak HJ, van Engelen BG \& Zwarts MJ. Muscle-fiber conduction velocity and electromyography as diagnostic tools in patients with suspected inflammatory myopathy: a prospective study. Muscle and Nerve 200429 46-50. (doi:10.1002/mus.10519)

38 Weber MA, Kinscherf R, Krakowski-Roosen H, Aulmann M, Renk H, Künkele A, Edler L, Kauczor HU \& Hildebrandt W. Myoglobin plasma level related to muscle mass and fiber composition: a clinical marker of muscle wasting? Journal of Molecular Medicine 200785 887-896. (doi:10.1007/s00109007-0220-3)
39 Merletti R, Farina D, Gazzoni M \& Schieroni MP. Effect of age on muscle functions investigated with surface electromyography. Muscle E Nerve 200225 65-76. (doi:10.1002/mus.10014)

40 Doherty TJ. Invited review: aging and sarcopenia. Journal of Applied Physiology 200395 1717-1727. (doi:10.1152/japplphysiol. $00347.2003)$

41 Inder WJ, Jang C, Obeyesekere VR \& Alford FP. Dexamethasone administration inhibits skeletal muscle expression of the androgen receptor and IGF-1 - implications for steroid-induced myopathy. Clinical Endocrinology 201073 126-132. (doi:10.1111/j.13652265.2009.03683.x)

Received 28 February 2011

Accepted 14 March 2011 